\title{
Anyons on a torus: Braid group, Aharonov-Bohm period, and numerical study
}

\author{
Yasuhiro Hatsugai and Mahito Kohmoto \\ Institute for Solid State Physics, University of Tokyo, 7-22-1 Roppongi, Minato-ku, Tokyo 106, Japan \\ Yong-Shi Wu \\ Department of Physics, University of Utah, Salt Lake City, Utah 84112
}

(Received 23 October 1990)

\begin{abstract}
We present a careful construction of anyons on a torus starting with the braid-group analysis. The rules of Wen, Dagotto, and Fradkin for putting anyons on a torus are reproduced with some minor improvements. The existence of noncontractible loops leads to braid-group representations characterized not only by anyon statistics $\theta$ but also by the magnetic fluxes $\Phi_{x}$ and $\Phi_{y}$ threading through the holes of the torus. The three parameters are tangled with each other. We explore the symmetries of the torus to separate the effects of $\Phi_{x}$ and $\Phi_{y}$ from those of $\theta$. It is shown that the anyon system always has a smaller period $\theta / \pi$ in $\Phi_{x}$ and $\Phi_{y}$ than the natural period 1. We perform several numerical calculations to investigate the spectral flow and consistency of the method and find interesting features in the spectral flow, which are relevant in understanding the fractional quantum Hall effect.
\end{abstract}

\section{INTRODUCTION}

Phase factors of wave functions present a delicate problem in quantum mechanics. Typical examples are the Aharonov-Bohm effect ${ }^{1}$ and Berry phase, ${ }^{2}$ where the phase of the wave function on a topologically nontrivial space plays a crucial role. In the path-integral formulation, the statistics phase factors characteristic of a manyparticle system originate from the nontrivial topology of the many-particle configuration space. ${ }^{3,4}$ They form a representation of the fundamental group of the latter. In three or higher dimensions, the allowed statistics is either Fermi or Bose statistics. In two dimensions, however, fractional statistics is allowed..$^{5-7}$ Particles obeying fractional statistics are called anyons. When two anyons are interchanged, the wave function of the system gets a complex phase $e^{i \theta}(\theta$ is the statistics parameter). This implies that the system breaks parity and time-reversal symmetries even in the absence of an external magnetic field.

The realization of fractional statistics is first recognized in the fractional quantum hall (FQH) effect where there is a strong magnetic field perpendicular to the twodimensional sample, ${ }^{8}$ as explicitly demonstrated by Arovas, Schrieffer, and Wilczek. ${ }^{9}$ The discovery of hightemperature superconductivity brings a renewed interest in strongly correlated electron systems. Recently, the possibility of realization of anyons was argued by Laughlin and co-workers ${ }^{10-12}$ who proposed that anyons play an important role in high- $T_{c}$ materials. It is argued that the doped holes in these materials behave as semions $(\theta=\pi / 2)$, and mean-field theory with random-phaseapproximation fluctuations predicts that the ground state of the anyon system is a superfluid. ${ }^{13-16}$ Although it is not clear yet whether anyons are relevant to real high- $T_{c}$ materials, the behavior of an anyon system is a very interesting problem to investigàte at least theoretically.
For a two-dimensional system, the fundamental group of the many-body configuration space is the braid group. The structure of the braid group crucially depends on the topology of the system. The planar case is the simplest, ${ }^{7}$ and the spherical case was first discussed by Thouless and $\mathrm{Wu}^{17}$ several years ago. Recently, Einarsson ${ }^{18}$ discussed the braid group on a torus and showed that fractional statistics (with $\theta \neq 0, \pi$ ) on a torus is consistent only with multicomponent wave functions (see also Ref. 19 for a Chern-Simons theory). There are studies of anyons on an annulus, ${ }^{20,21}$ and the present authors discussed the braid group on an annulus and a cylinder in a previous paper. ${ }^{22}$ The structure of the braid group determines the allowed statistics, ${ }^{17,18,22}$ and in some cases it determines the number of components of the anyon wave function. ${ }^{18}$ The braid-group representation also determines the period of spectral flow as a function of the flux threading through noncontractible loops. If we consider gauge invariance for fractionally charged excitations in an electronic system, the braid-group analysis leads to some strong restriction on the number of components of the effective wave function of the excitations. ${ }^{23}$

Wen, Dagotto, and Fradkin ${ }^{24}$ recently proposed how to put anyons on a torus. In the present paper, we explicitly construct anyons on a toroidal lattice by starting from the braid group. The resultant rules to define anyons are basically the same as those of Wen, Dagotto, and Fradkin, but our derivation from the first principles has the advantage of assuring their internal consistency, which was not rigorously proved in Ref. 24.

Similar to the situation on a cylinder, ${ }^{22}$ the existence of noncontractible loops on the torus leads to the appearance of additional generators in the braid group corresponding to moving an anyon along the noncontractible loops. This in turn requires more parameters to fix the braid-group representation, which can be identified as, in the case at hand, the central fluxes $\Phi_{x}$ and $\Phi_{y}$ threading 
through the two holes of the torus. How do the physical properties of the system depend on these fluxes? Using the braid-group analysis, we will show that in addition to the usual unit period for $\Phi_{x}$ and $\Phi_{y}$, for anyons on a torus with statistics $\theta$, there is a smaller period given by $\theta / \pi$. If $\theta=\pi p / q$ with $p$ and $q$ mutually prime, then the smallest period is actually $1 / q$ rather than 1 . So we are led to expect, for example, that the energy spectrum of anyons on a torus exhibits a period of $1 / q$ in $\Phi_{x}$ and $\Phi_{y}$. Since in general a given energy level does not necessarily have this period (it may have a larger period), we naturally expect the emergence of spectral flow or level crossing, in particular the rearrangements of energy levels as $\Phi_{x}$ and $\Phi_{y}$ change by $1 / q$. These expectations are confirmed by our numerical studies. The discovery of a smaller period in the central fluxes, which is closely related to the statistics of the anyons, is one of the main results of this paper.

With our explicit construction, we perform several numerical calculations to investigate the spectral flow and to confirm the consistency of the method. We find interesting features in the spectral flow which may have special importance in the FQH effect.

In Sec. II we first give a review of the relation of the braid group to many-body quantum mechanics and then derive several new results on the $\Phi_{x}\left(\Phi_{y}\right)$ dependence of, say, the energy spectrum of toroidal anyon system. In Sec. III we show the explicit construction (rule) of the anyons on the lattice with toroidal topology. The numerical results are shown in Sec. IV. Discussions and summary are given in Sec. $\mathrm{V}$.

\section{BRAID GROUP ON A TORUS}

Statistics in quantum mechanics can be clearly described by the path-integral formulation. ${ }^{4,5,7}$ The statistics is a weight (phase factor) in the path integral consistently assigned to each homotopy class of paths in the many-body configuration space. In this sense, statistics is determined by the representation of the first homotopy group (fundamental group) of the configuration space. In three and higher spatial dimensions, the fundamental group of the many-particle configuration space is a permutation group. Its one-dimensional representation corresponds to Fermi statistics or Bose statistics and the higher ones to parastatistics. ${ }^{25}$ The fundamental group of many-particle configuration space on a two-dimensional surface is the braid group. One-dimensional representation of the braid group yields fractional statistics. Namely, the scalar anyon wave function obeys the onedimensional representation of the braid group for the surface on which it is defined. For anyons on a torus, we have to use a representation whose dimension is larger than one. In this case the anyon wave functions must have more than one component. ${ }^{18}$

The braid group $B_{N}(S)$ for $N$ anyons on a surface $S$ is the first homotopy group of the $N$-anyon configuration space:

$$
C_{N}=(S \times \cdots \times S-D) / S_{N},
$$

where, because of the hard-core nature of anyons, one has to exclude the subset $D=\left\{\left(\mathbf{r}_{1}, \ldots, \mathbf{r}_{N}\right) \mid \mathbf{r}_{i}=\mathbf{r}_{j}, \exists i \neq j\right\}$ representing configurations with at least two of the particles located at the same site and $S_{N}$ is the permutation group of $N$ particles which represents their indistinguishability.

The braid group on a torus is discussed by Birman ${ }^{26}$ in detail. Its generators consist of $\widetilde{\sigma}_{j}, \widetilde{\rho}_{j}$, and $\widetilde{\tau}_{j}$. The local-exchange operator $\widetilde{\sigma}_{i}(i=1, \ldots, N-1)$ represents an interchange of $i$ th and $(i+1)$ th particle counterclockwise with no particles in the enclosed region. These generators are known to satisfy the following relations:

$$
\begin{aligned}
& \widetilde{\sigma}_{i} \widetilde{\sigma}_{j}=\widetilde{\sigma}_{j} \widetilde{\sigma}_{i} \quad(i \neq j \pm 1), \\
& \widetilde{\sigma}_{i} \widetilde{\sigma}_{i+1} \widetilde{\sigma}_{i}=\widetilde{\sigma}_{i+1} \widetilde{\sigma}_{i} \widetilde{\sigma}_{i+1} .
\end{aligned}
$$

On the torus there are two kinds of additional operators $\widetilde{\rho}_{j}$ and $\widetilde{\tau}_{j}(j=1, \ldots, N)$ which represent moving anyons along noncontractible loops as shown in Fig. 1. These generators satisfy

$$
\begin{aligned}
& \widetilde{\rho}_{j+1}=\widetilde{\sigma}_{j} \widetilde{\rho}_{j} \widetilde{\sigma}_{j}, \\
& \widetilde{\tau}_{j+1}=\widetilde{\sigma}_{j}^{-1} \widetilde{\tau}_{j} \widetilde{\sigma}_{j}^{-1} .
\end{aligned}
$$

(For the complete set of relations, see Birman. ${ }^{26}$ )

For $\widetilde{\sigma}_{j}$, the simplest representation is $\sigma_{j}=e^{i \theta}$ (for all $j$ ) as usual, and one can interpret $\theta$ as the anyon statistics. (If the braid-group representation is $M$ dimensional, this means $\sigma_{j}$ is $e^{i \theta}$ times the $M \times M$ unit matrix. Such representations describe the so-called scalar statistics in the cases with $M>1$.) Then (2.4) and (2.5) imply that the representations of $\widetilde{\rho}_{j}$ and $\widetilde{\tau}_{j}$ satisfy

$$
\begin{aligned}
& \rho_{j+1}=\rho_{j} \exp (i 2 \theta), \\
& \tau_{j+1}=\tau_{j} \exp (-i 2 \theta) .
\end{aligned}
$$

Using (2.6) and (2.7) repeatedly, one obtains

$$
\begin{aligned}
\rho_{j} & =\rho_{1} \exp [i 2 \theta(j-1)], \\
\tau_{j} & =\tau_{1} \exp [-i 2 \theta(j-1)] .
\end{aligned}
$$

To fix the braid-group representation, one needs to fix $\rho_{1}$ and $\tau_{1}$. This implies that the existence of the generators

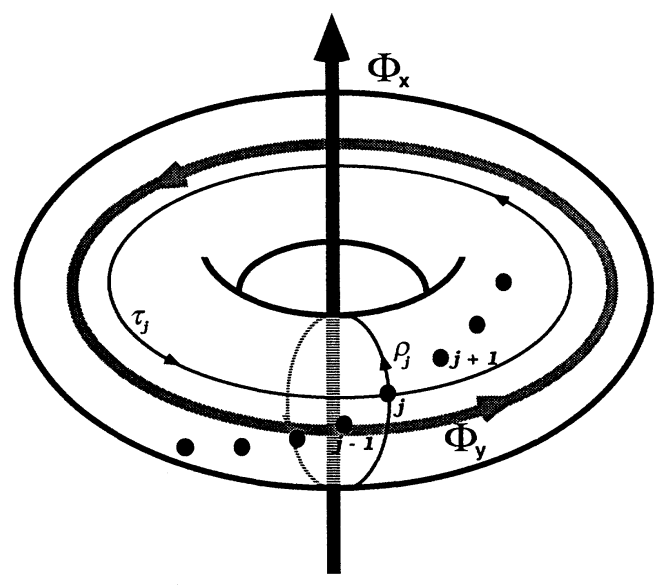

FIG. 1. Braid-group generators $\rho_{j}$ and $\tau_{j}$ on a torus. 
$\widetilde{\rho}_{j}$ and $\widetilde{\tau}_{j}$ requires additional parameters, in addition to $\theta$, to identify a braid-group representation on the torus. Physically, this is not surprising since the existence of the noncontractible loops makes it necessary to consider the Aharonov-Bohm effect, that is, the effects of central fluxes $\Phi_{x}$ and $\Phi_{y}$ threading through the holes (see Fig. 1). We include the effects of these fluxes by setting

$$
\begin{aligned}
& \tau_{1}=\exp \left(i 2 \pi \Phi_{x}\right) W_{x}, \\
& \rho_{1}=\exp \left(i 2 \pi \Phi_{y}\right) W_{y},
\end{aligned}
$$

where $W_{x}$ and $W_{y}$ are $M \times M$ matrices with $M$ being the dimension of the representation. Thus $\rho_{j}$ and $\tau_{j}$ are given by

$$
\begin{aligned}
\tau_{j} & =W_{x} \exp \left\{i\left[-2 \theta(j-1)+2 \pi \Phi_{x}\right]\right\}, \\
\rho_{j} & =W_{y} \exp \left\{i\left[2 \theta(j-1)+2 \pi \Phi_{y}\right]\right\} .
\end{aligned}
$$

Other useful relations for $\rho_{j}$ and $\tau_{j}$ are ${ }^{18,26}$

$$
\begin{gathered}
\tau_{i} \rho_{j}=\rho_{j} \tau_{i} e^{i 2 \theta} \quad(i \neq j), \\
\tau_{i} \rho_{i}=\rho_{i} \tau_{i} e^{-i 2 \theta(N-1)} .
\end{gathered}
$$

These relations together with (2.6) give

$$
\exp (i 2 \theta N)=1 \text {. }
$$

This is one of the conditions that relates the allowed values of $\theta$ to the total anyon number $N$. By taking a determinant of (2.14), one sees that $\theta$ is also restricted by

$$
\exp (i 2 \theta M)=1 \text {. }
$$

For an irreducible representation, $M$ must be the minimum integer which satisfies (2.17). If we set $\theta=\pi p / q$ with mutually prime integers $p$ and $q$, we obtain $M=q$.

To completely determine $W_{x}$ and $W_{y}$, let us consider symmetries of the torus. If $\Phi_{x}=\Phi_{y}=0$, there is the $x-y$ symmetry; that is, the system is invariant if one exchanges $x$ and $y$ axes. This implies that we can transform $W_{x}$ to $W_{y}$ by a unitary transformation so they are unitarily equivalent. Thus the sets of the eigenvalues of $W_{x}$ and $W_{y}$ should be the same. Also, (2.14) and (2.15) give a relation between $W_{x}$ and $W_{y}$ as

$$
W_{x} W_{y}=W_{y} W_{x} e^{i 2 \theta}
$$

To satisfy these relations, in an appropriate basis, $W_{x}$ and $W_{y}$ must be of the following form:

$$
\begin{aligned}
& W_{x}=e^{i \xi}\left[\begin{array}{cccc}
0 & 1 & & \\
\vdots & 0 & \ddots & \\
0 & & \ddots & 1 \\
1 & 0 & \cdots & 0
\end{array}\right], \\
& W_{y}=e^{i \xi}\left[\begin{array}{cccc}
c^{1+\eta} & 0 & & \\
0 & c^{2+\eta} & & \\
& & \ddots & 0 \\
& & 0 & c^{q+\eta}
\end{array}\right],
\end{aligned}
$$

where $c=e^{i 2 \theta}$ and $\eta$ is an arbitrary integer. The origin of the physical flux is determined by $\xi$. The torus has a rotational symmetry in the three-dimensional space which reverses the direction of $\Phi_{x}$ and $\Phi_{y}$. By the same rotation, the orientations of the loops which represent $\rho_{j}$ and $\tau_{j}$ also get reversed. Note that the effects of $\sigma_{j}$ 's are unchanged by the operation. So we require the following two equivalences:

$$
\begin{aligned}
& \tau_{j}\left(\Phi_{x}\right) \simeq \tau_{N-j+1}^{-1}\left(-\Phi_{x}\right), \\
& \rho_{j}\left(\Phi_{y}\right) \simeq \rho_{N-j+1}^{-1}\left(-\Phi_{y}\right) .
\end{aligned}
$$

From (2.20) and (2.21), we get

$$
\begin{aligned}
& c W_{x} \simeq W_{x}^{-1}, \\
& c^{-1} W_{y} \simeq W_{y}^{-1} .
\end{aligned}
$$

The equivalence requires that the two matrices have the same set of eigenvalues. The eigenvalues of $W_{x}$ and $W_{y}$ are given by $\left\{e^{i \xi} c^{j} \mid j=1, \ldots, q\right\}$. Since $c$ is given by $c=e^{i 2 \theta}=\exp (i 2 \pi p / q)$, we have $c W_{x} \simeq W_{x}$ and $c^{-1} W_{y} \simeq W_{y}$. Also $\left\{c^{j} \mid j=1, \ldots, q\right\}$ is equal to $\left\{c^{-j} \mid j=1, \ldots, q\right\}$ as a set. Thus we can get the equality for the two sets from (2.22) and (2.23):

$$
\begin{aligned}
\left\{e^{i \xi} c^{1}, e^{i \xi} c^{2}, \ldots\right. & \left., e^{i \xi} c^{q}\right\} \\
& =\left\{e^{-i \xi} c^{1}, e^{-i \xi} c^{2}, \ldots, e^{-i \xi} c^{q}\right\} .
\end{aligned}
$$

This implies that

$$
\xi=0 \text { or } \frac{\pi}{q}\left(\bmod \frac{2 \pi}{q}\right) \text {. }
$$

We cannot further resolve this ambiguity by this consideration. In the numerical calculations below, we take $\xi=0$. The form of (2.19) has been obtained previously in Ref. 18 , but the constraint (2.25) on $\xi$ is a new result of this paper.

In the expression of $W_{y}$ in (2.19), we can get an equivalent representation by taking a different $\eta$. This means that changing $\eta$ is a gauge transformation, and it does not affect the physical quantities. To change $\eta$ to $\eta+1$ corresponds to changing $\Phi_{y}$ to $\Phi_{y}+\theta / \pi$. This means the period of the flux is reduced to $1 / q$ [recall that the original period for the phase factors in (2.10) and (2.11) is 1]. Thus all the physical quantities are periodic functions of $\Phi_{x}$ and $\Phi_{y}$ with a period $1 / q$. For example, an eigenenergy $E_{n}$ must satisfy

$$
\begin{aligned}
E_{n}\left(\Phi_{x}, \Phi_{y}\right) & =E_{n}\left[\Phi_{x}+\frac{1}{q}, \Phi_{y}\right) \\
& =E_{n}\left[\Phi_{x}, \Phi_{y}+\frac{1}{q}\right]
\end{aligned}
$$

The existence of a smaller period in $\Phi_{x}$ and $\Phi_{y}$ than the natural period of one looks a bit surprising at first glance. Here we give another proof for this result which makes it physically more understandable. Geometrically, because of the periodic boundary conditions on a torus, there is intrinsically not any difference between the two loops corresponding to, say, $\widetilde{\rho}_{j}$ and $\widetilde{\rho}_{j+1}$. However, their 
representation matrices $\rho_{j}$ and $\rho_{j+1}$ are not identical, but related to each other by (2.6). The only way that (2.6) can be consistent with the geometrical periodicity mentioned above is that $\rho_{j}$ and $\rho_{j+1}$ are unitarily equivalent to each other; this can be directly verified for the explicit expression of $\rho_{j}$ given by (2.13) and (2.19). On the other hand, (2.13) implies that if we shift $\Phi_{y}$ by an amount $\theta / \pi$, then $\rho_{j}$ changes into $\rho_{j+1}$ and we obtain an equivalent representation, which describes exactly the same system. Therefore, any physical property of the anyon system is a function periodic in $\Phi_{y}$ with period $1 / q$ if $\theta=\pi p / q$. In Sec. IV numerical results will be shown to confirm the existence of this small period.

When we consider the ground-state energy, there is another symmetry. As discussed later,the Hamiltonian of the system depends on $\tau_{j}\left(\Phi_{x}\right)$ and $\rho_{j}\left(\Phi_{y}\right)$. The Schrödinger equation is

$$
H\left(\Phi_{x}, \Phi_{y}\right) \Psi\left(\Phi_{x}, \Phi_{y}\right)=E\left(\Phi_{x}, \Phi_{y}\right) \Psi\left(\Phi_{x}, \Phi_{y}\right)
$$

The dependence on $\Phi_{x}$ and $\Phi_{y}$ is due to that of $\tau_{j}\left(\Phi_{x}\right)$ and $\rho_{j}\left(\Phi_{y}\right)$. Let us take a complex conjugate of $\tau_{j}\left(\Phi_{x}\right)$ and $\rho_{j}\left(\Phi_{y}\right)$ in (2.12) and (2.13). We can also show that $W_{x}^{*} \simeq W_{x}$ and $W_{y}^{*} \simeq W_{y}$ both for $\xi=0$ and $\pi / q$. Thus we have

$$
H\left(\Phi_{x}, \Phi_{y}\right)^{*}=H\left(-\Phi_{x},-\Phi_{y}\right)
$$

If we take a complex conjugate of (2.27) and consider that $E\left(\Phi_{x}, \Phi_{y}\right)$ is real, (2.28) means that

$$
E\left(\Phi_{x}, \Phi_{y}\right)=E\left(-\Phi_{x},-\Phi_{y}\right) .
$$

Again, this is consistent with the rotational symmetry of the torus in three-space which reverses the sign of both $\Phi_{x}$ and $\Phi_{y}$.

\section{PUTTING ANYONS ON A TORUS}

To investigate anyons on a torus numerically, one has to be able to define anyons on a two-dimensional lattice (we consider an $L_{x} \times L_{y}$ lattice) with periodic boundary conditions for both $x$ and $y$ directions. The guiding principle for doing this is to produce necessary phase factors for all noncontractible loops in the many-body configuration space which are consistent with the braidgroup representation. Wen, Dagotto, and Fradkin ${ }^{24}$ proposed a set of rules for putting anyons on a torus by clever guesswork and performed several consistency checks. But they were not able to prove rigorously the internal consistency of their rules. Our construction, while giving basically the same rules as theirs (with some minor improvements), clarifies the origin of the rules from the braid group point of view. This not only makes some peculiarities understandable, but also provides a theoretical proof for the internal consistency. We have previously constructed several sets of rules to define anyons on a lattice with annulus or cylinder topology. ${ }^{21}$ The idea is to combine two different sets of rules on the annulus for noncontractible loops in the $x$ and $y$ directions of the torus.

\section{A. Hamiltonian and $M$-component basis}

The Hamiltonian of the anyon system is given by

$$
H=-\sum_{\langle i j\rangle} c_{j}^{\dagger} e^{i \theta_{i j}} c_{i}+\text { H.c. }
$$

where the summation is over the nearest neighbors and $c_{i}$ is the hard-core boson operator at site $i,{ }^{14}$ satisfying

$$
\begin{aligned}
& {\left[c_{i}, c_{j}^{\dagger}\right]=\delta_{i j},} \\
& c_{i}^{\dagger} c_{i}^{\dagger}=0 .
\end{aligned}
$$

The phase factor $\theta_{i j}=-\theta_{j i}$ is defined on the link $\langle i, j\rangle$, and it is determined by the string rule explained in the following subsection. Here we may identify $\theta_{i j}$ as

$$
\frac{2 \pi e^{*}}{c h} \int_{1}^{j} \mathbf{A} \cdot \mathrm{dl},
$$

where $\mathbf{A}$ is a total vector potential; the quantity

$$
\begin{aligned}
\frac{1}{2 \pi} \sum_{\text {around } S} \theta_{i j} & =\frac{e^{*}}{c h} \oint_{\partial S} \mathbf{A} \cdot \mathbf{d} \mathbf{l} \\
& =\frac{1}{\Phi_{0}^{*}} \int_{S} \mathbf{B} \cdot \mathbf{d} \mathbf{S}
\end{aligned}
$$

is the total (statistical plus real) magnetic flux through the area $S$ in units of the flux quantum $\Phi_{0}^{*}=\frac{c h}{e^{*}}$. In these expression $e^{*}$ is the charge of anyons. In Secs. II, III, and IV, we take $\Phi_{0}^{*}=1$.

The Hilbert space of the system is spanned by the basis with base vectors labeled by the positions of the particles and with an extra component index

$$
\left|\left\{\mathbf{r}_{1}, \mathbf{r}_{2}, \ldots, \mathbf{r}_{N}\right\}, k\right\rangle \quad(k=1,2, \ldots, M),
$$

where $\mathbf{r}_{j}$ is the two-dimensional coordinate vector of the $j$ th particle and $k$ specifies the component. Here the order of $\mathbf{r}_{j}$ is irrelevant in this basis. For a one-component wave function (used in the annulus or cylinder geometry), the basis is just given by $\left|\left\{\mathbf{r}_{1}, \mathbf{r}_{2}, \ldots, \mathbf{r}_{N}\right\}\right\rangle$. We may interpret (3.4) as $M$ copies of one-component bases. We may call the index $k$ as a sheet index in this sense. From (3.1) we can determine hopping matrix elements between the base vectors (3.4).

\section{B. Explicit construction of the string rule}

Since the rule for incorporating the fluxes $\Phi_{x}$ and $\Phi_{y}$ is well-known, here we only discuss the rules with $\Phi_{x}=\Phi_{y}=0$. We define the anyon system by an appropriate assignment of the phases $\theta_{i j}$, which should be consistent with the braid-group representation we discussed before. Such an assignment of $\theta_{i j}$ can be obtained by assigning strings as follows. Assign to each anyon located at, say, the site $(m, n)$ a vertical $\theta$ string from the adjoining plaquette $\left(m+\frac{1}{2}+\delta n, n-\frac{1}{2}\right)$ to $\left(m+\frac{1}{2}+\delta n, \frac{1}{2}\right)$ where it is turned into a horizontal $2 \theta$ string which always ends at the one and same point $\left(L_{x}+\frac{1}{2}, \frac{1}{2}\right)$ as shown in Fig. 2. Here $\delta$ is an infinitely small positive number to avoid the ambiguity in the positions of the vertical strings 


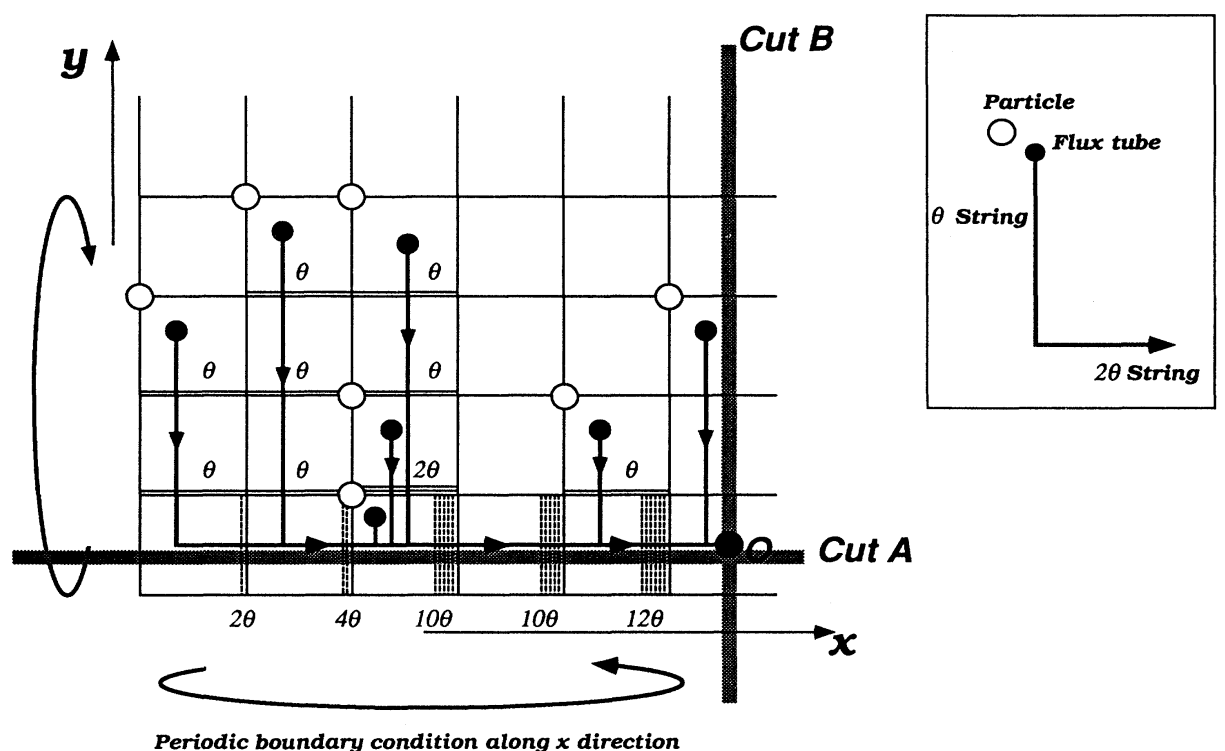

FIG. 2. String rule to define anyons on a toroidal lattice which satisfies the requirement of the braid-group representation.

when several particles have the same $x$ coordinate. If an anyon hops from left to right across such a vertical $\theta$ string, the change in phase is given by $e^{+i \theta}$; the hopping in the opposite direction across the string would give rise to an opposite phase $e^{-i \theta}$. If a $\theta$ string sweeps an anyon, we determine the phase factor as if the anyon crosses the $\theta$ string. If an anyon hops upward across a $2 \theta$ string, the change in phase is given by $e^{+i 2 \theta}$, and if it hops downward, the change in phase is given by $e^{-i 2 \theta}$. In addition to giving $\sigma_{j}=e^{i \theta}$, these $\theta$ and $2 \theta$ strings are designed so as to satisfy (2.6) and (2.7).

To realize an $M$-dimensional representation for $\widetilde{\rho}_{j}$ and $\widetilde{\tau}_{j}$ fully, we have to put two cuts as shown in Fig. 2. First, we consider the generators $\widetilde{\rho}_{j}$ 's. The representation for $\widetilde{\rho}_{j}$ is diagonal as shown in (2.13) and (2.19). This implies that when an anyon hops across the cut $A$ upward, the sheet index of the wave function does not change, but the phase change depends on the sheet index $k$ of the state and is given by $c^{k+\eta}=e^{i 2 \theta(k+\eta)}$. Thus we assign to the cut $A$ the phase factor

$$
\exp [i 2 \theta(k+\eta)] \text {. }
$$

We have to note that there is an important correction for hopping in the $y$ direction across the horizontal boundary at $y=L_{y}+\frac{1}{2}$. In this case, we have to add an additional phase factor $e^{i \theta\left(X_{m}-1\right)}$, where $X_{m}$ is the number of particles which have the same $x$ coordinate $m$ as the particle that goes across the boundary. Necessity of this correction originates from the ordering the vertical $\theta$ strings of the particles having the same $x$ coordinate according to their $y$ coordinates, exactly the same as in the annulus case discussed in our previous paper. ${ }^{22}$ This correction rule improves that of Wen, Dagotto, and Fradkin (Fig. 11 in Ref. 24).

Next, let us consider the generators $\widetilde{\tau}_{j}$ 's. The representation for $\widetilde{\tau}_{j}$ shown in (2.12) and (2.19) is not diagonal in the sheet index. In accordance to the form of the matrix $W_{x}$ shown in (2.19), we require that when the particle hops across the cut $B$ from left to right, the index $k$ decreases from $k$ to $k-1$. Furthermore, we assign $e^{i(N-1) \theta}$ to such hopping to compensate an unphysical contribution to $\Phi_{x}$ from anyon fluxes. This point is explained in detail in our previous paper ${ }^{22}$ where this correction is used to define "rule B" for the anyon system on an annulus. (This correction is not mentioned in Ref. 24.) The effects of the cut $B$ are

$$
\begin{aligned}
& \mid k(\text { sheet index })\rangle \rightarrow|k-1\rangle, \\
& \exp [i(N-1) \theta] .
\end{aligned}
$$

From these rules on the torus, it seems that there is a finite flux tube at the end point $O$ of the strings (see Fig. 2 ). One can, however, check that this is not so. Consider moving a particle around $O$ along a clockwise loop as shown in Fig. 3. We assume the original sheet index is $k$.

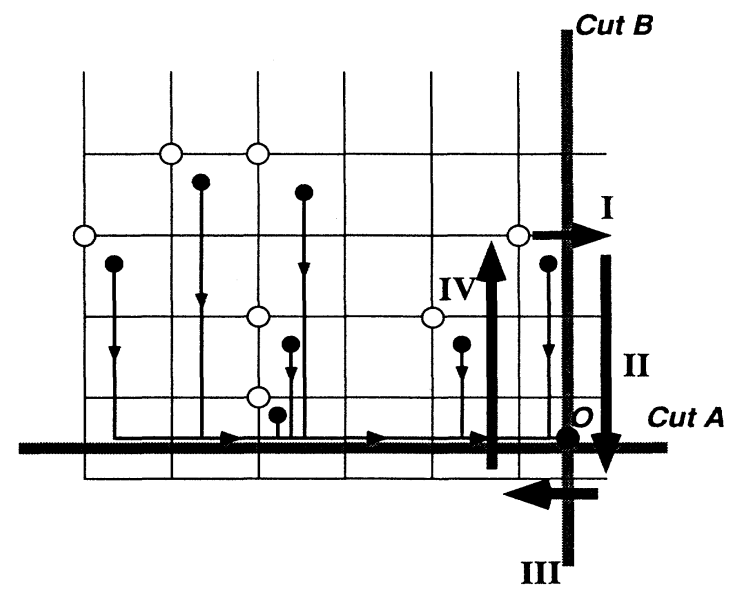

FIG. 3. Effect of the end point of the strings. 
In process I the change in the phase is $e^{i \theta(N-1)}$ and the sheet index becomes $k-1$. In process II the phase change is $e^{-i 2 \theta(k+\eta-1)}$ because the sheet index is decreased to $k-1$. In process III the sheet index returns to $k$ and the phase change is $e^{-i \theta(N-1)}$. In the last process IV, the phase change is $e^{i 2 \theta(k+\eta)} \times e^{i 2 \theta(N-1)}$, where the first factor comes from cut $A$ and the second factor comes from the $2 \theta$ strings of the remaining particles. Thus there is no net change in sheet index and the total change of the phase is $e^{i 2 \theta N}$, which is 1 due to (2.16).

\section{NUMERICAL RESULTS}

We treat an $L_{x} \times L_{y}$ finite-size system with $N$ particles by an exact diagonalization and denote it by $L_{x} \times L_{y} / N$. For a small system, we use the usual packages of a combination of the Hauseholder method (to get eigenvalues) and the bisection method (to get eigenvectors). We can get all the states for a small system such as $3 \times 3 / 3$. For a larger lattice, we also use the Lanczos method to obtain eigen energies. We calculate not only the ground-state energies, but also excited energies as a function of $\Phi_{x}$ and $\Phi_{y}$.

Write $\theta=\pi p / q$, where $p$ and $q$ are mutually prime integers. We calculate many cases with different choices of $\theta$ and $N$. Here we present some typical examples. In Fig. 4 we set $\Phi_{y}$ to be zero and show the energies as a function of $\Phi_{x}$ (a) for $\pi \frac{1}{3}$ statistics in the $3 \times 3 / 3$ system, (b) for $\pi \frac{1}{2}$ statistics (semion) in the $3 \times 3 / 4$ system, and (c) for $\pi \frac{2}{7}$ statistics in the $3 \times 3 / 7$ system. They show that the spectrum is indeed invariant if we change $\Phi_{x}$ to $\Phi_{x}+1 / q$. The period of each level, however, is generally neither $1 / q$ nor 1 . in some cases [Figs. 4(a) and 4(b)], several levels are separated from the other levels; that is, there is an energy gap between a set of low-lying states and others. One can see that there are always at least $q$ equivalent states which transform into each other when $\Phi_{x}$ changes to $\Phi_{x}+n / q$. The degeneracy of each level is at most 2 , and we do not observe the $q$-fold degeneracy claimed by Wen and $\mathrm{Niu}^{27}$ from an effective theory of the FQH effect on the torus. Presumably, the $q$-fold degeneracy is lifted by the finite-size effect discussed by them.

We show the ground-state energy versus $\left(\Phi_{x}, \Phi_{y}\right)$ plot in Fig. 5. The energy diagram is symmetric under exchanging $\Phi_{x}$ and $\Phi_{y}$ or under reversing the sign of both. It is consistent to the braid-group consideration in Sec. III.

\section{SUMMARY AND DISCUSSIONS}

In this paper we have presented a careful construction of anyons on a torus starting from the braid-group analysis. Our main concern is the relationship to the braid group on the torus and the topological features arising from the existence of noncontractible loops.

To define a representation of the braid group on the torus, we have to include the central fluxes $\Phi_{x}$ and $\Phi_{y}$. A braid-group representation is characterized by three parameters $\left(\theta, \Phi_{x}, \Phi_{y}\right)$, and they are tangled with each other. We have explored the symmetries of the torus to (a)

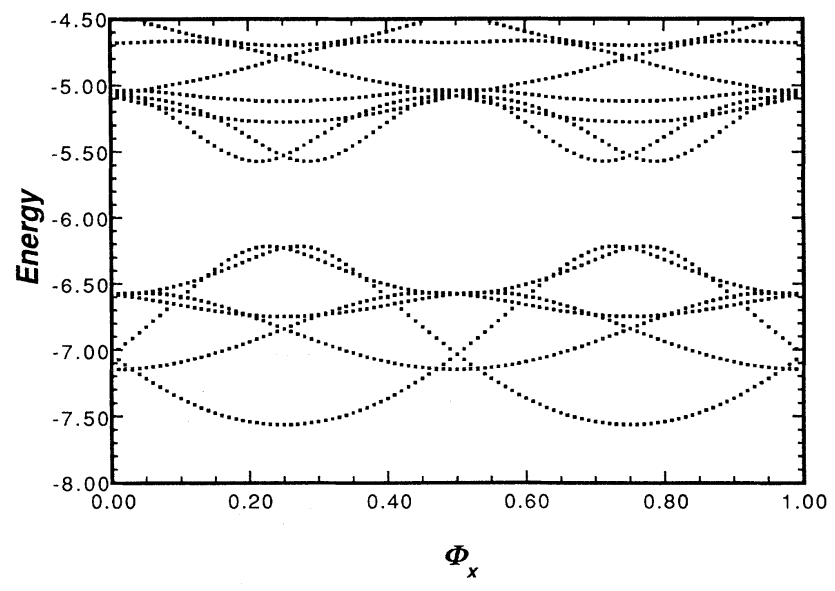

(b)

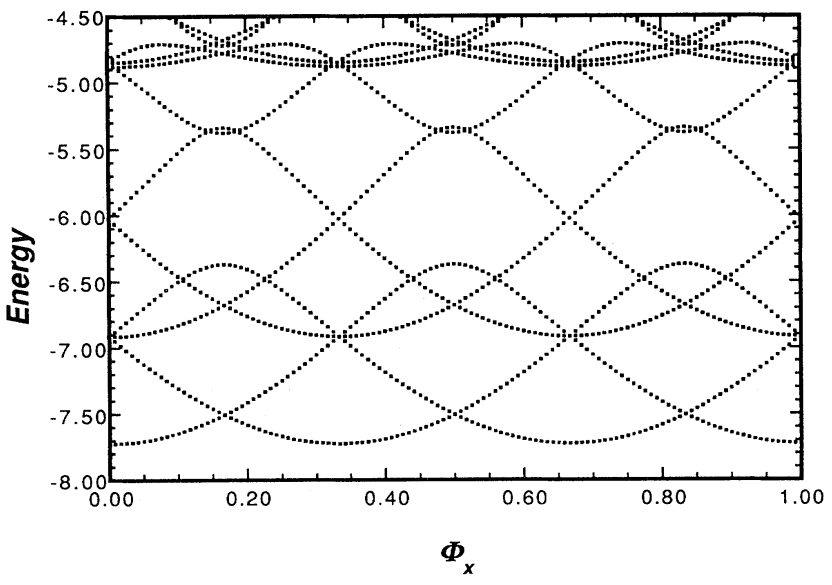

(c)

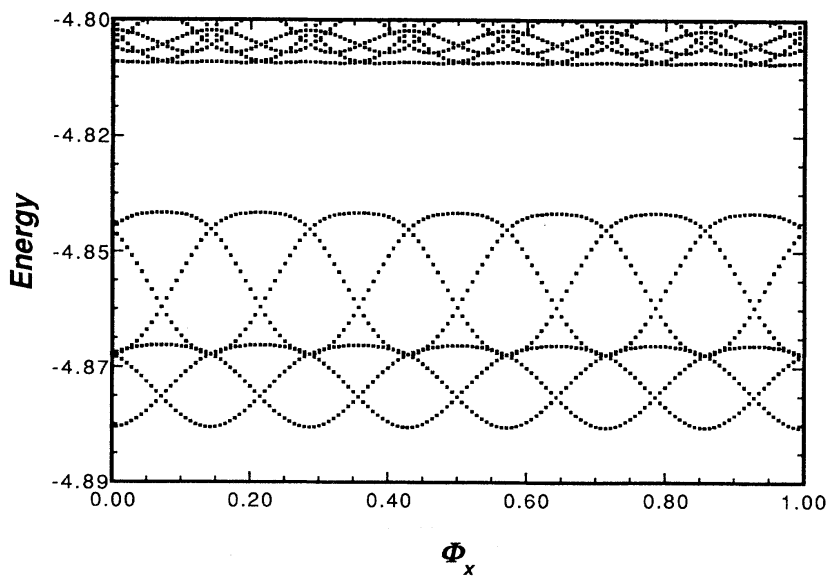

FIG. 4. Spectral flow in anyon systems with (a) $3 \times 3 / 3$ and $\theta=\pi / 3$, (b) $3 \times 3 / 4$ and $\theta=\pi / 2$ (semion), and (c) $3 \times 3 / 7$ and $\theta=2 \pi / 7$. 


\section{Spectral Flow}

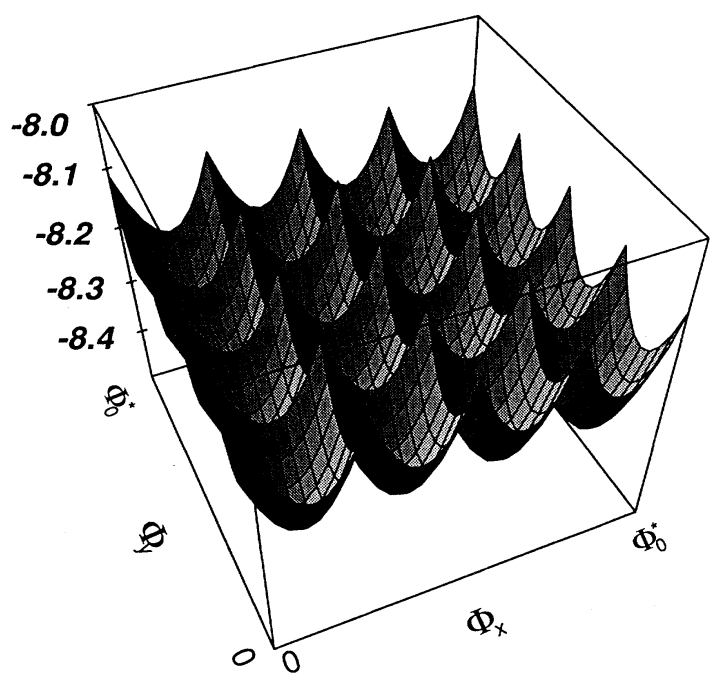

FIG. 5. Spectral flow in $3 \times 3 / 4$ and $\theta=\pi / 4$ anyon system as a function of $\left(\Phi_{x}, \Phi_{y}\right)$. It shows that there are symmetries: $E\left(\Phi_{x}, \Phi_{y}\right)=E\left(-\Phi_{x}, \Phi_{y}\right)=E\left(\Phi_{x},-\Phi_{y}\right)=E\left(\Phi_{y}, \Phi_{x}\right)$.

separate the effects of the physical flux $\Phi_{x}$ and $\Phi_{y}$ from those of $\theta$. We also conclude that the physical observables are invariant under changing both signs of $\Phi_{x}$ and $\Phi_{y}$. Though our Eq. (2.29) is consistent with the general consideration of Byers and Yang, ${ }^{28}$ the argument for it is different. They have explored the time-reversal symmetry which is no longer true for anyons. Instead, we exploited the rotational symmetry of the toroidal geometry in three-space.

Let us explicitly introduce the flux quantum $\Phi_{0}^{*}=h c / e^{*}$ in the following. We have calculated the spectral flow for several small systems. The spectrum returns to itself when we change $\Phi_{x}$ to $\Phi_{x}+(p / q) \Phi_{0}^{*}$ because of the new gauge invariance of the representation of the braid group under changing $\eta$ in (2.19). Physical$1 y$, this can be easily understood, at least in the limiting case in which hopping matrix elements across the boundary at $\left(L_{x}, j_{y}\right)-\left(1, j_{y}\right)$ are infinitesimally small. In this case, the effect of $\tau_{j}$ 's is negligible and the system is essentially $q$ decoupled copies of an annulus. The $q$ annuluses have different twisted boundary conditions specified by $W_{y}$ in (2.19); that is, the $k$ th sheet feels a $2 \theta(k+\eta)$ extra flux through the hole of the annulus. This means that the origins of the $\Phi_{x}$ for the $k$ th and $(k+1)$ th annuluses differ by $\Phi_{0}^{*} p / q$ and explains why, at least in this limit, the system has an invariance under changing $\Phi_{x}$ by $\Phi_{0}^{*} / q$.

Furthermore, we note that the ground states we obtained from our numerical calculation are not always $q$ fold degenerate, but this is not in conflict with the expectation that the ground state is at least $q$-fold degenerate by an effective-field-theoretical argument. ${ }^{27}$ This is because our results are for finite anyon systems, but the effective-field-theory argument is applicable only in the thermodynamic limit. To define the thermodynamic limit, we have to treat a fixed-density system; that is, we have to treat more particles when we consider a larger system and consider the energy per particle. In this limit, it is natural to expect the effects of the twisted boundary condition become negligible. This implies that there is no spectrum flow for energy per particle [the $(E / N)-\Phi$ diagram becomes horizontal lines] and the $q$ sheets described above should give the same energy per particle; that is, the ground state is $q$-fold degenerate in the thermodynamic limit.

Finally, let us consider the relation of our results to the fractional quantum Hall (FQH) effect. When the Landau level filling is $v=1 / q$, it is widely believed that excitations of the system are quasiparticles which have fractional charge $e^{*}=e / q$ and statistics $\theta=\pi 1 / q .{ }^{8,9,29,30}$ Our consideration discussed above holds correct even if there is a strong magnetic field. There are two types of gauge invariance in this system. The period $\Phi_{0}^{*}$ implies the gauge invariance of the fractionally charged quasiparticle. We note that in this case the period $\Phi_{0}^{*} / q=\Phi_{0}$ coincides with that for gauge invariance of the constituent particle (the electron). Otherwise, we have to introduce other excitation to recover the gauge invariance. We have discussed the situation in detail for the annulus system in previous article. ${ }^{23}$

Using the argument that there are two kinds of gauge invariances, we can discuss the FQH effect in the way of Laughlin $^{29}$ and Halperin ${ }^{30}$ for the integral case. Our consideration has been for a torus; however, we may treat an annulus as a limiting case of it as discussed above. An argument for the FQH effect by the gauge invariance for electrons was proposed by $\mathrm{TaO}$ and $\mathrm{Wu}^{31}$ and refined by Niu, Thouless, and $\mathrm{Wu}^{32}$ and by Thouless. ${ }^{33} \mathrm{We}$ follow this argument from the anyon's point of view. It is known that the quasiparticles in the $\mathrm{FQH}$ system with the filling $v=1 / q$ are anyons with $e^{*}=e / q$ and $\theta=\pi / q .^{9}$ Let us assume that the Fermi energy lies in a gap caused by the strong magnetic field. The FQH system has the property that when we increase the central flux by $\Delta \Phi=\Phi_{0}^{*}$ [with $\Phi_{0}^{*}$ determined by the anyon (quasiparticle) charge $\left.e^{*}\right\}$, the system returns to its original state. Since $\Phi_{0}^{*}$ is exactly an integral multiple ( $q$ times) of the period $\Phi_{0}$ determined by the electron charge $\left(\Phi_{0}=h c / e\right)$, the topological arguments presented in Ref. 32 guarantee that exactly $n$ electrons are transported across the potential difference $V_{x}$. (Note that the transported particles are not anyons but electrons because the anyons are only quasiparticles in the FQH effect.) Thus the change in energy is given by $\Delta E=n e V_{x}$. We use a formula for the current by Byers and Yang: ${ }^{28}$

$$
I_{y}=c \frac{\Delta E}{\Delta \Phi}
$$

It implies that Hall conductance may be fractional as

$$
\sigma_{x y}=\frac{n}{q} \frac{e^{2}}{h}
$$




\section{ACKNOWLEDGMENTS}

Y.S.W. acknowledges financial support from the Japan Society for Promotion of Sciences and the warm hospital- ity at the Institute for Solid State Physics, University of Tokyo, which made this collaboration possible. His work was supported in part by U.S. National Science Foundation Grant No. 8706501.
${ }^{1}$ Y. Aharonov and D. Bohm, Phys. Rev. 115, 485 (1959).

${ }^{2}$ M. V. Berry, Proc. Soc. London, Ser. A 392, 45 (1984).

${ }^{3}$ M. G. G. Laidlaw and C. M. Dewitt, Phys. Rev. D 3, 1275 (1975).

${ }^{4}$ L. S. Schulman, Techniques and Applications of Path Integration (Wiley, New York, 1981).

5J. M. Leinaas and J. Myrheim, Nuovo Cimento B 37, 1 (1977).

${ }^{6}$ F. Wilczek, Phys. Rev. Lett 48, 1144 (1982); 49, 957 (1982).

${ }^{7}$ Y. S. Wu, Phys. Rev. Lett. 52, 2103 (1984); 53, 111 (1984).

${ }^{8}$ See, e.g., The Quantum Hall Effect, edited by R. E. Prange and S. M. Girvin (Springer-Verlag, New York, 1987).

${ }^{9}$ D. Arovas, J. R. Schrieffer, and F. Wilczek, Phys. Rev. Lett. 53, 722 (1984).

${ }^{10}$ V. Kalmeyer and R. B. Laughlin, Phys. Rev. Lett. 59, 2095 (1987).

${ }^{11}$ R. B. Lauglin, Phys. Rev. Lett. 60, 2677 (1988).

${ }^{12}$ R. B. Laughlin, Science 242, 525 (1988).

${ }^{13}$ A. L. Fetter, C. B. Hanna, and R. B. Laughlin, Phys. Rev. B 39, 9679 (1989).

${ }^{14}$ C. B. Hanna, R. B. Laughlin, and A. L. Fetter, Phys. Rev. B 40, 8745 (1989).

${ }^{15}$ Y. -H. Chen, F. Wilczek, E. Witten, and B.I. Halperin, Int. J. Mod. Phys. B 3, 1001 (1989).

${ }^{16}$ Y. Kitazawa and H. Murayama, Phys. Rev. B 41, 11101 (1990).
${ }^{17}$ D. J. Thouless and Y. S. Wu, Phys. Rev. B 31, 1191 (1985).

${ }^{18}$ T. Einarsson, Phys. Rev. Lett. 64, 1995 (1990).

${ }^{19}$ Y. Hosotani, Phys. Rev. Lett. 622785 (1989).

${ }^{20}$ G. S. Canright, S. M. Girvin, and A. Brass, Phys. Rev. Lett. 63, 2291 (1989).

${ }^{21}$ G. S. Canright, S. M. Girvin, and A. Brass, Phys. Rev. Lett. 63, 2295 (1989).

${ }^{22}$ Y. Hatsugai, M. Kohmoto, and Y. S. Wu, Phys. Rev. B 43, 2661 (1991).

${ }^{23}$ Y. S. Wu, Y. Hatsugai, and M. Kohmoto, Phys. Rev. Lett. 66, 659 (1991).

${ }^{24}$ X. G. Wen, E. Daggoto, and E. Fradkin, Phys. Rev. B 42, 6110 (1990).

${ }^{25}$ Y. Ohnuki and S. Kamefuchi, Quantum Field Theory and Parastatistics (Tokyo University Press, Tokyo, 1982).

${ }^{26}$ J. S. Birman, Commun. Pure Appl. Math. 22, 41 (1969).

${ }^{27}$ X. G. Wen and Q. Niu, Phys. Rev. B 41, 9377 (1990).

${ }^{28}$ N. Byers and C. N. Yang, Phys. Rev. Lett. 7, 46 (1961).

${ }^{29}$ R. B. Laughlin, Phys. Rev. B 23, 5632 (1981).

${ }^{30}$ B. I. Halperin, Phys. Rev. Lett. 30, 1583 (1984).

${ }^{31}$ R. Tao and Y. S. Wu, Phys. Rev. B 31, 6859 (1985).

${ }^{32}$ Q. Niu, D. J. Thouless, and Y. S. Wu, Phys. Rev. B 31, 3372 (1985).

${ }^{33}$ D. Thouless, Phys. Rev. B 40, 12034 (1989). 